\title{
Subglottic haemangioma
}

\author{
C A Sherrington, D K Y Sim, N J Freezer, C F Robertson
}

\begin{abstract}
Objectives-To describe experience with subglottic haemangioma in a unit where conservative treatment has been favoured.

Methods-Retrospective case note review of infants presenting with subglottic haemangioma over a 25 year period.

Results-Thirty one infants were identified. Diagnosis was difficult where skin haemangiomas were absent and where the lesion was circumferential rather than asymmetrical. Tracheostomy was safe, well tolerated, and managed by the patients' family at home. Major complications were seen only when laser treatment was used.

Conclusions-Aggressive treatment with substantial risks of long term complications may not be necessary in this spontaneously resolving disorder.

(Arch Dis Child 1997;76:458-459)
\end{abstract}

Keywords: subglottic haemangioma; tracheostomy

Subglottic haemangiomas are rare vascular malformations that enlarge from shortly after birth until 8-18 months of age, when spontaneous involution begins. Complete regression usually occurs by $5-8$ years of age. ${ }^{1}$ Numerous treatment modalities have been advocated, most commonly tracheostomy, ${ }^{2}$ systemic steroids, ${ }^{3}$ carbon dioxide laser, ${ }^{45}$ or interferon alfa-2a. ${ }^{1}$ Any physically destructive treatment may lead to permanent subglottic stenosis.

\section{Patients and methods}

The patient records of all children presenting with subglottic haemangioma at the Royal Children's Hospital, Melbourne in the 25 years between 1 January 1970 and 1 January 1995 were reviewed. Subglottic haemangioma was diagnosed by the typical clinical history and bronchoscopic appearance without a biopsy sample being taken.

Data are presented as median (range) values. Appropriate non-parametric statistical analyses were used with significance if $\mathrm{p}<0.05$.

Department of

Thoracic Medicine, Royal Children's Hospital, Parkville, Victoria 3052, Australia

C A Sherrington

D K Y Sim

N J Freezer

C F Robertson

Correspondence to: Dr Sherrington.

Accepted 15 January 1997 from birth. Only one child had an onset symptoms after 3 months of age. Prematurity was noted in six infants.
Skin haemangiomas were present in 20 infants $(65 \%)$ and were noted at birth in nine $(29 \%)$. They affected the head or neck region in $17(55 \%)$ infants and solely the peripheral regions in three $(10 \%)$. Their presence was associated with more accurate clinical and bronchoscopic diagnosis (table 1) and a longer duration of tracheostomy (575 $v 295$ days; $\mathrm{p}=$ 0.05). Abnormalities apart from subglottic haemangioma were present in three infants and included aortic and other vascular dysplasia, midline sternal defect, and cleft lip and palate.

Subglottic haemangioma was reported in the differential diagnosis in only $15(48 \%)$ infants before bronchoscopy. The correct diagnosis was made during the first admission in only 21 $(68 \%)$ infants. Of the $10(32 \%)$ infants with an incorrect diagnosis, four (with skin haemangiomas) did not undergo bronchoscopy during the first admission, whereas six others had an incorrect bronchoscopic diagnosis. Circumferential lesions were present in a total of seven infants (of 23 infants with these data) and in four of six infants with an incorrect bronchoscopic diagnosis.

The median occlusion of the tracheal cross sectional area was $70 \%(5-90 \%)$. The degree of occlusion did not predict the need for tracheostomy nor correlate with the duration of cannulation required. Subglottic haemangioma showed minor extension to other contiguous sections of the airway in only three infants.

Table 2 summarises the treatment modalities used. Tracheostomy was required in $27(87 \%)$ infants and was performed during the first hospital admission in 18 (58\%) infants. Cannulation was required for a median of 455 days (range 25-1441 days). Decannulation was successful in all instances. There were no significant perioperative nor long term complications of tracheostomy and no significant airway problems after decannulation. Surgical treatment was required in six $(19 \%)$ infants for the removal of an airway granuloma and in 10 (32\%) infants for the closure of a persistent tracheal fistula after decannulation.

Between 1978 and 1988 carbon dioxide laser ablation was used in nine children aged over 18 months for a median of four times each (range 2-15 times). Subglottic stenosis was seen in two infants with four and 15 laser procedures. Spontaneous resolution of the stenosis occurred after 1441 and 1248 days of tracheal cannulation, respectively.

Steroids were used in seven (23\%) infants, with a definite clinical response in two $(6 \%)$ (who avoided tracheostomy). Interferon alfa-2a was used in two infants, with clear success in one. Both infants showed mild neutropenia 
Table 1 Effect of the presence of skin haemangiomas on diagnosis

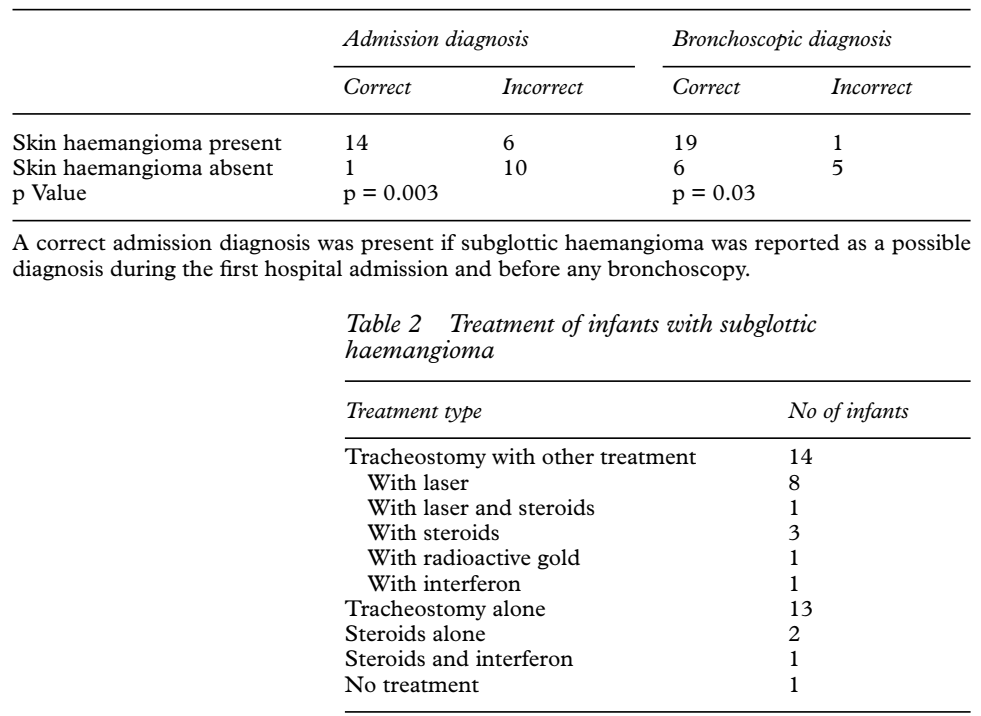

and alterations in serum aspartate transaminase levels.

\section{Discussion}

This study confirms the clinical features of subglottic haemangioma reported in other series. ${ }^{2-6}$ Symptoms may be present at birth and almost always begin before 4 months of age. Diagnosis may be difficult owing to the rarity of the lesion and the similarity of presentation with other more common illnesses. The presence of a skin haemangioma may alert the doctor to the correct clinical and bronchoscopic diagnosis.

If needed in the management of subglottic haemangioma, a tracheostomy is well tolerated in small children with few complications and is safely managed by the patient's family at home. ${ }^{7}$ The temporary nature of any associated morbidity differentiates it from all other invasive forms of treatment.

Local destruction is only effective when the lesion is confined to the tracheal submucosa.
Laser treatment avoids the need for tracheostomy in approximately $90 \%$ of infants and might decrease the cannulation time, but is associated with subglottic stenosis in approximately $20 \%$ of infants, particularly those with larger lesions. ${ }^{45}$

Steroids have been widely used. The rebound of symptoms is common with decreasing dosage and implies a need for prolonged treatment. Interferon alfa-2a appears to be an effective systemic drug, acting by interference with angiogenesis. Its side effects are generally not serious, most commonly fever, abnormalities in liver function tests, and neutropenia. ${ }^{1}$

Any treatment aimed at decreasing the size of the lesion must be judged against the natural history of spontaneous involution over time, leaving a normal larynx. Our current practice is to use systemic steroids or interferon for small lesions where the airway is adequate, in an attempt to avoid tracheostomy. Where the airway is not adequate, a tracheostomy is performed and a trial of interferon is used in an attempt to decrease the period of cannulation. More aggressive treatment with a substantial risk for long term sequelae may not be necessary in this essentially self limiting disorder.

1 Ohlms LA, Jones DT, McGill TJ, Healy GB. Interferon alfa-2a therapy for airway hemangiomas. Ann Otol Rhinol Laryngol 1994;103:1-8

2 Williams HE, Phelan PD, Stocks JG, Wood H. Haemangioma of the larynx in infants: diagnosis, respiratory mechanics and management. Aust Paediatr f 1969;5:14954.

3 Kveton JF, Pillsbury HC. Conservative treatment of infantile subglottic hemangioma with corticosteroids. Arch Otolaryngol 1982;108:117-9.

4 Healy G, McGill T, Friedman EM. Carbon dioxide laser in subglottic hemangioma. An update. Ann Otol Rhinol Laryngol 1984;93:370-3.

5 Sie KC, McGill T, Healy GB. Subglottic hemangioma: ten years' experience with the carbon dioxide laser. Ann Otol Rhinol Laryngol 1994;103:167-72.

6 Shikhani AH, Jones MM, Marsh BR, Holliday MJ. Infantile subglottic hemangiomas. An update. Ann Otol Rhinol Laryngol 1986;95:336-47.

7 Freezer NJ, Beasley SW, Robertson CF. Tracheostomy. Arch Dis Child 1990;65:123-6. 